\title{
RESEARCH
}

Open Access

\section{Identification of potential blood biomarkers for early diagnosis of Alzheimer's disease through RNA sequencing analysis}

Daichi Shigemizu ${ }^{1,2,3^{*+}} \mathbb{D}$, Taiki Mori ${ }^{1}$, Shintaro Akiyama', Sayuri Higaki ${ }^{1}$, Hiroshi Watanabe', Takashi Sakurai ${ }^{4,5}$, Shumpei Niida ${ }^{1}$ and Kouichi Ozaki ${ }^{1,3+}$

\begin{abstract}
Background: With demographic shifts toward older populations, the number of people with dementia is steadily increasing. Alzheimer's disease (AD) is the most common cause of dementia, and no curative treatment is available. The current best strategy is to delay disease progression and to practice early intervention to reduce the number of patients that ultimately develop AD. Therefore, promising novel biomarkers for early diagnosis are urgently required.

Methods: To identify blood-based biomarkers for early diagnosis of AD, we performed RNA sequencing (RNA-seq) analysis of 610 blood samples, representing 271 patients with AD, 91 cognitively normal (CN) adults, and 248 subjects with mild cognitive impairment $(\mathrm{MCl})$. We first estimated cell-type proportions among $A D, M C l$, and $C N$ samples from the bulk RNA-seq data using CIBERSORT and then examined the differentially expressed genes (DEGs) between $A D$ and $C N$ samples. To gain further insight into the biological functions of the DEGs, we performed gene set enrichment analysis (GSEA) and network-based meta-analysis.

Results: In the cell-type distribution analysis, we found a significant association between the proportion of neutrophils and $A D$ prognosis at a false discovery rate $(F D R)<0.05$. Furthermore, a similar trend emerged in the results of routine blood tests from a large number of samples ( $n=3,099: \mathrm{AD}, 1,605 ; \mathrm{MCl}, 994 ; \mathrm{CN}, 500)$. In addition, GSEA and network-based meta-analysis based on DEGs between AD and CN samples revealed functional modules and important hub genes associated with the pathogenesis of AD. The risk prediction model constructed by using the proportion of neutrophils and the most important hub genes (EEF2 and RPL7) achieved a high AUC of 0.878 in a validation cohort; when further applied to a prospective cohort, the model achieved a high accuracy of 0.727 .

Conclusions: Our model was demonstrated to be effective in prospective AD risk prediction. These findings indicate the discovery of potential biomarkers for early diagnosis of $A D$, and their further improvement may lead to future practical clinical use.
\end{abstract}

Keywords: Alzheimer's disease, RNA sequencing, Biomarkers for early diagnosis

\footnotetext{
* Correspondence: d.shigemizu@gmail.com

${ }^{\dagger}$ Daichi Shigemizu and Kouichi Ozaki contributed equally to this work.

'Medical Genome Center, National Center for Geriatrics and Gerontology,

7-430 Morioka-cho, Obu 474-8511, Aichi, Japan

${ }^{2}$ Department of Medical Science Mathematics, Medical Research Institute,

Tokyo Medical and Dental University (TMDU), Tokyo 113-8510, Japan

Full list of author information is available at the end of the article
}

C C The Author(s). 2020 Open Access This article is licensed under a Creative Commons Attribution 4.0 International License, which permits use, sharing, adaptation, distribution and reproduction in any medium or format, as long as you give appropriate credit to the original author(s) and the source, provide a link to the Creative Commons licence, and indicate if changes were made. The images or other third party material in this article are included in the article's Creative Commons licence, unless indicated otherwise in a credit line to the material. If material is not included in the article's Creative Commons licence and your intended use is not permitted by statutory regulation or exceeds the permitted use, you will need to obtain permission directly from the copyright holder. To view a copy of this licence, visit http://creativecommons.org/licenses/by/4.0/ The Creative Commons Public Domain Dedication waiver (http://creativecommons.org/publicdomain/zero/1.0/) applies to the data made available in this article, unless otherwise stated in a credit line to the data. 


\section{Background}

With demographic shifts toward older populations, the number of people with dementia is steadily increasing. The total number of people with dementia worldwide has been estimated to be 75 million by 2030 and 135 million by 2050 [1]. Since there is no treatment or prevention for $\mathrm{AD}$, the current best strategy is to delay disease progression and to practice early intervention to reduce the number of patients that ultimately develop AD [2]. Therefore, promising novel biomarkers for early diagnosis are urgently required $[3,4]$.

Alzheimer's disease (AD) is the most common cause of dementia, accounting for 60 to $80 \%$ of dementia cases [5]. Genome-wide association studies (GWAS) have identified several genetic factors that contribute to $\mathrm{AD}$ risk [6-8]. However, the cause of the disease still remains to be elucidated. The current AD diagnosis is generally based on assessing patients' cognitive function. These examinations are not performed routinely, because they are time-consuming and the results largely depend on the physician's experience [9, 10]. Alternatively, cerebrospinal fluid (CSF) biomarkers, including amyloid-beta 1-42 (A $\left.\beta_{1-42}\right)$, total tau (T-tau), and phosphorylated tau $181\left(\mathrm{P}-\mathrm{tau}_{181}\right)$ [11, 12], and positron emission tomography (PET) imaging scans [13-15] are effective for $\mathrm{AD}$ diagnosis, but because of the highly invasive nature of CSF collection and high cost of PET, using these biomarkers as part of a general physical examination to facilitate early diagnosis and therapeutic intervention remains challenging.

Compared with CSF biomarkers and PET imaging scans, blood-based biomarkers are attractive as affordable alternatives for the diagnosis of AD. Mattsson et al. recently reported that plasma neurofilament light level (NfL) has the potential to be a noninvasive biomarker to monitor neurodegeneration in AD [16]. Janelidze et al. reported that plasma $\mathrm{P}-\mathrm{tau}_{181}$ is a noninvasive diagnostic and prognostic biomarker of $\mathrm{AD}$ [17]. One of the most powerful tools for detecting those biomarkers, whole RNA sequencing (RNA-seq) of human peripheral blood mononuclear cells (PBMCs) by using a next-generation sequencer, is widely applied and supports comprehensive analysis of the entire transcriptome [18-20]. The most important application of the RNA-seq data analysis is the identification of differentially expressed genes (DEGs) [21-23]. Systems biology analyses using DEGs reveal key functional modules and important hub genes associated with the pathogenesis of diseases (e.g., Gene Ontology [GO] [24, 25], Kyoto Encyclopedia of Genes and Genomes [KEGG] biological pathways [26, 27]). However, to our knowledge, no previous studies have involved comprehensive RNA-seq analysis of a large number of $\mathrm{AD}$ samples and applied an mRNA-based risk prediction model to a prospective cohort.
Here, we performed large-scale RNA-seq transcriptome analyses on a large number of AD samples to detect potential blood-based biomarkers for earlier diagnosis of AD. To this end, we used the RNA-seq data to evaluate cell-type composition among samples from subjects with $\mathrm{AD}$, mild cognitive impairment (MCI), and normal cognitive function $(\mathrm{CN})$ and to compare DEGs between $\mathrm{AD}$ and $\mathrm{CN}$ samples. Subsequent gene set enrichment analyses (GSEA) and network-based meta-analysis using the DEGs revealed new potential biomarkers for $\mathrm{AD}$ diagnosis. The risk prediction model using those potential biomarkers achieved a high AUC in a validation cohort and effectively determined $A D$ risk in a prospective cohort. We believe that, once optimized, these new potential biomarkers will be of practical clinical use in the early diagnosis of AD.

\section{Methods}

\section{Sample collection}

All of the 610 subjects whose blood samples were evaluated for mRNA expression and their associated clinical data were obtained from the National Center for Geriatrics and Gerontology (NCGG) Biobank, which collects human biomaterials and data for geriatrics research. Of them, 271 subjects were AD patients, 91 subjects were elderly $\mathrm{CN}$ controls, and 248 patients had mild cognitive impairment $(\mathrm{MCI})$. All of the subjects were 60 years or older (Supplementary Table S1). The AD and MCI subjects were diagnosed with probable or possible $A D$ according to the criteria of the National Institute on Aging Alzheimer's Association workgroups [9, 10]. Patients with probable $\mathrm{AD}$ were used as $\mathrm{AD}$ subjects in this study. The $\mathrm{CN}$ subjects had subjective cognitive abnormalities but normal cognition on a neuropsychological assessment, which included a comprehensive neuropsychological test, Mini-Mental State Examination (MMSE) score $>27$. All of the 3,099 subjects $(1,605$ ADs, $994 \mathrm{MCIs}$, and $500 \mathrm{CNs}$ ) with the proportion of neutrophils measured in routine blood tests were also obtained from the NCGG Biobank. All of these subjects were also $\geq 60$ years in age (Supplementary Table S2).

\section{cDNA library preparation and RNA sequencing}

Buffy coat samples were isolated from the whole blood according to the standard operating procedure of NCGG Biobank [28]. Buffy coat fractions containing leukocytes were separated by centrifugation $(3,500 \mathrm{rpm}, 5 \mathrm{~min}, \mathrm{RT})$ and were frozen for further use. Total RNAs in buffy coat samples were isolated using the miRNeasy Mini Kit (Qiagen, Hilden, Germany) according to the manufacturer's instructions with slight modification. TRIzol LS reagent $(1 \mathrm{~mL})$ (Thermo Fisher Scientific, MA, USA) and 1-bromo-3-chloropropane $(100 \mu \mathrm{L})$ (Tokyo Chemical Industry, Tokyo, Japan) were added to each sample. Samples were mixed thoroughly by shaking for more 
than $30 \mathrm{~s}$ and incubated at room temperature for $3 \mathrm{~min}$. Phase separation was performed by centrifugation at 15 , $000 \mathrm{~g}$ at $4{ }^{\circ} \mathrm{C}$ for $15 \mathrm{~min}$. The upper aqueous phase was collected and loaded into the miRNeasy mini-column. After washing, total RNAs were extracted by RNase free water $(50 \mu \mathrm{L})$. Only high-quality samples with an RNA integrity number (RIN) value $\geq 6.0$ were used to construct the sequencing library (Supplementary Table S1). Sequencing libraries were prepared by using $500 \mu \mathrm{g}$ of total RNA for each sample with Illumina TruSeq Stranded Total RNA with Ribo-Zero Globin and IDT for Illumina-TruSeq UD Indexes according to the manufacturer's instructions (Illumina, San Diego, CA). The libraries were subsequently sequenced by using Illumina NovaSeq6000 platform with paired-end reads of $151 \mathrm{bp}$ according to the manufacturer's instructions.

\section{RNA sequencing data analysis}

The quality of the read sequences (fastq files) was assessed by using FastQC (version 0.11.7). The lowquality reads $(<\mathrm{Q} 20)$ and trimmed reads with adaptor sequences (shorter than $50 \mathrm{bp}$ ) were discarded by using Cutadapt (version 1.16). The remaining clean sequenced reads were mapped to the human reference genome (GRCh37) by using STAR [29] (2-pass option, version 2.5.2b). By using the featureCounts program [30] from the subread package (version 1.6.6), read counts for each gene were calculated to generate expression levels. Outlier read counts (i.e., the top and bottom $5 \%$ of read counts for each gene) were replaced as the maximum and minimum of the remaining effectives, respectively. The read counts from each sample were then combined into a count file, on which differential expression analysis was performed by using edgeR [31] (version 3.18.1). Genes with a threshold CPM (counts per million reads mapped) $>1$ in more than onefourth of all sequenced samples were used for further analysis. The caclNormFactorsfunction in edgeR [31] was used to obtain a trimmed mean of $M$ value normalization factors to account for library sizes. Dispersion was calculated by using the estimateCommonDisp and estimateTagwiseDisp functions in edgeR [31]. The exactTest function in edgeR [31] was applied to obtain DEGs between AD and $\mathrm{CN}$ samples.

\section{Proportions of immune cell types according to bulk RNA sequencing data}

After RNA-seq reads were aligned to the human reference genome by using STAR [29], RSEM [32] (version 1.3.0) was used to quantify transcripts per million (TPM), which were suitable for use with CIBERSORT [33] (version 1.0.1). While CIBERSORT estimated the proportions of 22 immune cell types, we recategorized these 22 cell types into 12 major cell types by summing the proportions as appropriate. The 12 cell types we evaluated were (1) B cells (naive and memory), (2) plasma cells, (3) $\mathrm{CD}^{+} \mathrm{T}$ cells, (4) $\mathrm{CD} 4^{+} \mathrm{T}$ cells $\left(\mathrm{CD} 4^{+} \mathrm{T}\right.$ cells naive, memory resting, and memory activated; $\mathrm{T}$ cells follicular helper; and T cells regulatory), (5) $\gamma \delta \mathrm{T}$ cells, (6) NK cells (resting and activated), (7) monocytes, (8) macrophages (M0, M1, and M2), (9) dendritic cells (resting and activated), (10) mast cells (resting and activated), (11) eosinophils, and (12) neutrophils.

\section{In silico biological and functional analysis}

Gene Ontology (GO) [24, 25] classification, which is comprised of three major categories-biological process, cellular component, and molecular function-is useful for uncovering the functions of genes of interest. The DAVID [25, 34] (version 6.8) gene functional classification tool (https://david.ncifcrf.gov) was used to generate annotations. DAVID was applied to a list of differentially expressed genes with FDR $<0.05$ and fold change $>1.2$, and statistically significant GO terms and KEGG biological pathways were identified. Statistically significant GO terms were further expressed as a $z$-score (the number of upregulated genes minus the number of downregulated genes divided by the square root of the count) and presented in a circular visualization by using the GOplot package (version 1.0.2) in R [35].

\section{Network-based meta-analysis}

Network-based analysis was performed by using NetworkAnalyst [36] with the STRING Interactome database [37], which provides comprehensive information regarding interactions between proteins, including prediction and experimental interaction data. The confidence cutoff score was set to 700 . The protein-protein interaction (PPI) network was constructed by using zero-order interaction network analysis (direct interaction only) and graphically generated by using Cytoscape v3.7.1 (http://www.cytoscape.org/) [38].

\section{Risk prediction model construction}

RNA-seq data were split: two-thirds were used for a training data set and one-third for a test data set. Using the training data, we constructed risk prediction models based on clinical information (age, sex, and $A P O E \varepsilon_{4}$ genotypes), the proportion of neutrophils, and the topranked $p$ hub genes using a random forest classifier. The top-ranked $p$ hub genes were then selected stepwise $(p=1,2, \ldots 10)$. The optimal hyper-parameters in the training data were determined by using 10 -fold crossvalidation. The adjusted model was then evaluated on the test data, which were completely independent of the training data, by using AUC as the discriminative accuracy of the risk prediction model. The method used in this study was implemented through the caret package (version 6.0.76) in R (https://www.r-project.org/). 
qRT-PCR validation of gene expression

cDNA was synthesized by using a PrimeScriptII 1st Strand cDNA Synthesis Kit (Takara Bio, Shiga, Japan). Quantitative RT-PCR (qRT-PCR) analysis was performed by using customized TaqMan gene expression assays (Applied Biosystems, Waltham, MA) and the Quantstudio7 Flex Real-Time PCR System (Thermo Fisher, Waltham, MA). The following commercially available TaqMan gene expression assays were used: EEF2 (Hs00157330_m1), RPL7 (Hs02596927_g1), LDHB (Hs00929956_m1), NR1D2 (Hs00233309_m1), PDK4 (Hs01037712_m1), TRIOBP (Hs00980819_m1), TAS2R39 (Hs00603443_s1), BASP1 (Hs00932356_s1), and ACTB (Hs01060665_g1). The qRT-PCR conditions were as follows: one cycle of $50^{\circ} \mathrm{C}$ for $2 \mathrm{~min}$ and $95^{\circ} \mathrm{C}$ for $20 \mathrm{~s}$ followed by 40 cycles of $95^{\circ} \mathrm{C}$ for $1 \mathrm{~s}, 60^{\circ} \mathrm{C}$ for $20 \mathrm{~s}$, and $72{ }^{\circ} \mathrm{C}$ for $30 \mathrm{~s}$. Each gene was assayed in duplicate. $A C T B$ was pre-selected as a reference gene for normalization of target gene expression levels. Gene expression levels from qRT-PCR were calculated relative to the reference gene $A C T B$ using the semi-quantitative method [39]. The gene expressions were obtained for $10 \mathrm{AD}$ and $10 \mathrm{CN}$ randomly selected samples. The $\log 2$ fold change $(\log \mathrm{FC})$ was obtained from the average values of the gene expressions.

\section{Results}

\section{RNA sequencing data}

A total of 610 samples, comprising $271 \mathrm{AD}, 248 \mathrm{MCI}$, and $91 \mathrm{CN}$ samples, were enrolled in this study (Table 1). Using a high-throughput next-generation system to perform RNA sequencing (RNA-seq) analysis, we obtained an average of 44.3, 47.3, and 43.9 million raw read sequences from the $\mathrm{AD}, \mathrm{MCI}$, and $\mathrm{CN}$ samples, respectively, of which $99.6 \%, 99.5 \%$, and $99.6 \%$ were highquality (i.e., > Q20) read sequences. After low-quality read sequences were discarded and reads with adaptor sequences were trimmed, 43.8, 47.3, and 43.2 million reads of cleaned data remained for the $\mathrm{AD}, \mathrm{MCI}$, and $\mathrm{CN}$ samples, respectively, of which $82.7 \%, 82.1 \%$, and $82.1 \%$ uniquely mapped to the human reference genome (GRCh37) (Supplementary Table S3).

\section{Comparison of cell-type distribution among $A D, M C l$, and CN samples}

To detect blood-based biomarkers, we first used the bulk RNA-seq data to compare cell-type distribution among $\mathrm{AD}, \mathrm{MCI}$, and $\mathrm{CN}$ samples. Specifically, CIBERSORT [33] estimated the relative proportions (as transcripts per million [TPM]) of 12 major types of immune cells (i.e., B cells, plasma cells, $\mathrm{CD}^{+}{ }^{\mathrm{T}}$ cells, $\mathrm{CD} 4^{+} \mathrm{T}$ cells, $\gamma \delta$ $\mathrm{T}$ cells, NK cells, monocytes, macrophages, dendritic cells, mast cells, eosinophils, and neutrophils) in each sample. We used the Jonckheere-Terpstra trend test to identify a statistically significant increase or decrease in cell-type proportion among $\mathrm{AD}, \mathrm{MCI}$, and $\mathrm{CN}$ samples. Accordingly, the proportion of neutrophils was significantly increased in AD prognosis at an FDR $<0.05$ (neutrophils, 0.007; Fig. 1a and Supplementary Table S1). The proportions of $\mathrm{B}$ cells and $\gamma \delta \mathrm{T}$ cells also showed significant differences in $\mathrm{AD}$ prognosis at an $\mathrm{FDR}<0.05$ (B cells, 0.019; $\gamma \delta$ T cells, 0.007; Fig. 1a and Supplementary Table S1), but these proportions were very low in all samples and too difficult to determine if they were truly associated with the AD prognosis.

To further investigate the association between an increased neutrophil count and AD prognosis, we used a larger number of samples $(n=3,099$ : $\mathrm{AD}, 1,605$; MCI, 994; and $\mathrm{CN}, 500$ ) to examine the neutrophil population determined through routine blood tests. Interestingly, these data sets obtained by using routine blood tests revealed the same increase in the neutrophil proportion as the RNA-seq data $(P=0.002$, Jonckheere-Terpstra trend test; Fig. $1 \mathrm{~b}$ and Supplementary Table S2). Therefore, these results provided strong evidence that an increased neutrophil proportion might be useful as a blood-based biomarker for the diagnosis of $\mathrm{AD}$. The proportion of neutrophils estimated from RNA-seq data was positively correlated with that calculated through routine blood tests (254 ADs, 232 MCIs, and 85 CNs; Pearson $r=0.56$, $P<0.01$, Fig. 1c). We also performed a principal component analysis with RNA-seq data of the three groups, but we could not observe the significant difference among the three (Fig. 1d).

Table 1 Summary of characteristics for $A D, M C l$, and $C N$ samples

\begin{tabular}{|c|c|c|c|}
\hline Characteristic & $A D$ & $\mathrm{MCl}$ & $\mathrm{CN}$ \\
\hline Sample number & 271 & 248 & 91 \\
\hline Male:female & $1: 2.15$ & $1: 1.30$ & $1: 0.82$ \\
\hline $\begin{array}{l}\text { Age (mean } \pm 1 \\
\text { S.D.) }\end{array}$ & $79.55 \pm 5.83$ & $77.37 \pm 6.12$ & $71.29 \pm 5.07$ \\
\hline $\begin{array}{l}\text { MMSE (mean } \pm \\
\text { S.D.) }\end{array}$ & $18.09 \pm 4.49$ & $24.54 \pm 2.98$ & $29.32 \pm 0.94$ \\
\hline $\begin{array}{l}\text { APOE } \\
\text { genotypes }\end{array}$ & $\begin{array}{l}E 2 / 2=2, E 3 / 2=14, E 3 / 3=148, E 4 / 2=3, E 4 / 3= \\
88, E 4 / 4=16\end{array}$ & $\begin{array}{l}E 2 / 2=1, E 3 / 2=11, E 3 / 3=163, E 4 / 2=1, E 4 / 3= \\
60, E 4 / 4=12\end{array}$ & $\begin{array}{l}\mathrm{E} 3 / 2=5, E 3 / 3=73, E 4 / 3=12, \\
E 4 / 4=1\end{array}$ \\
\hline
\end{tabular}

MMSE Mini-Mental State Examination (a comprehensive neuropsychological test) 


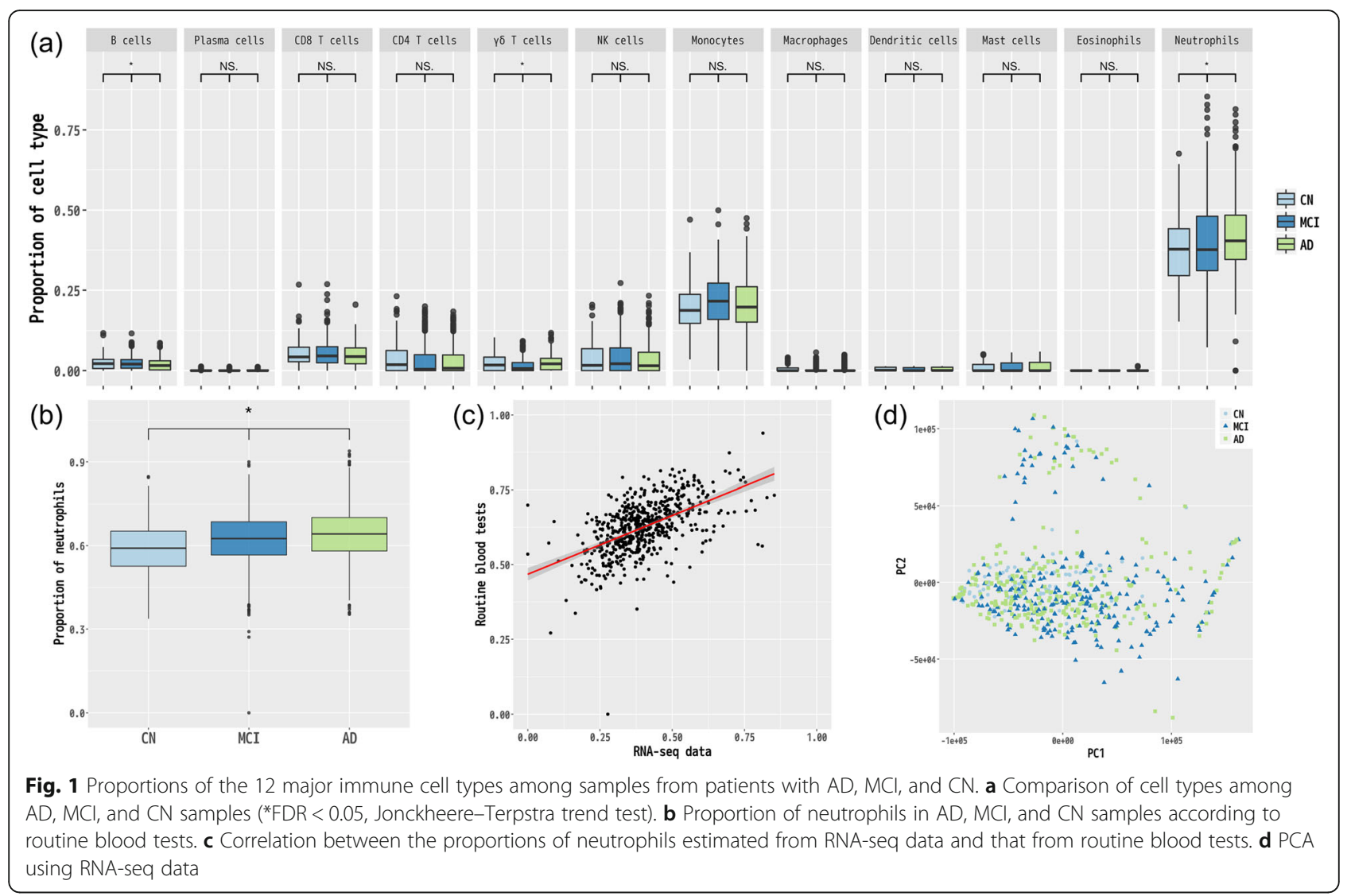

(a)

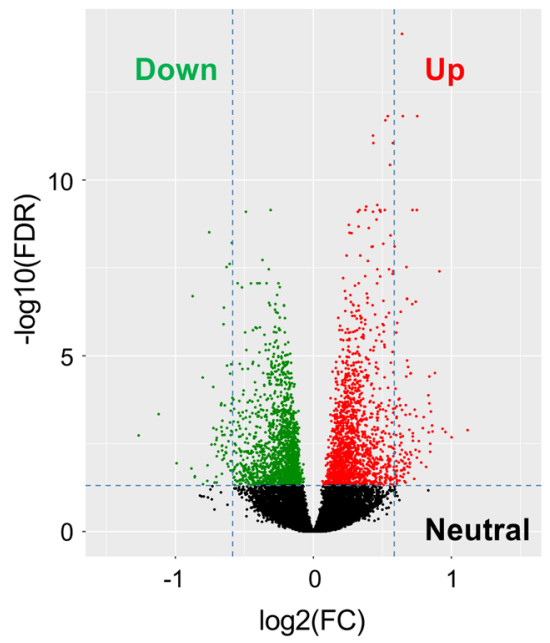

(b)
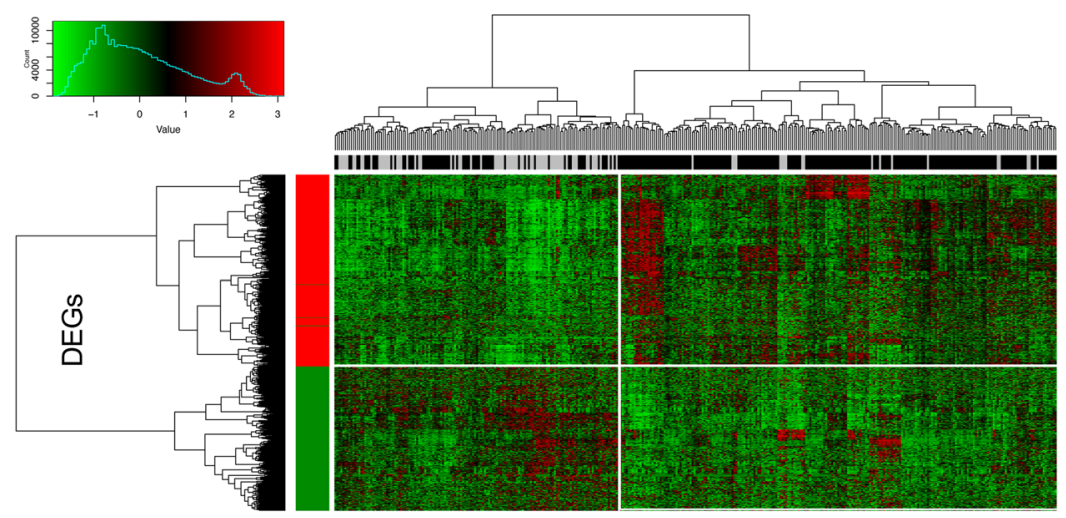

Samples

Fig. 2 Distribution of differentially expressed genes (DEGs). a Each point represents a gene. Green and red dots represent downregulated and upregulated DEGs, respectively. $\mathbf{b}$ Hieratical clustering of DEGs and samples by using the trimmed mean of M-values normalization factors. The horizontal and vertical axes represent the samples (AD, black; CN, gray) and DEGs (red, upregulated; green, downregulated), respectively 


\section{Detection of DEGs}

Focusing on the 19,699 genes with a threshold of $>1$ CPM (counts per million reads mapped) in more than one-fourth of all sequenced samples, we next examined the DEGs in AD and $\mathrm{CN}$ samples. A total of 846 statistically significant DEGs (i.e., FDR $<0.05$ and fold change $>1.2$ ) with Entrez gene IDs were identified, of which 480 genes were upregulated and 366 were downregulated in the AD samples (Fig. 2a and Supplementary Table S4). In addition, a heatmap of DEGs using the trimmed mean of $M$ value normalization factors showed that the expression profiles of the $\mathrm{AD}$ and $\mathrm{CN}$ samples clustered separately (Fig. 2b).

\section{Biological and functional analysis}

To gain further insight into the biological functions of the DEGs, we performed a gene set enrichment analysis (GSEA) using the DAVID (version 6.8) gene functional classification tool (https://david.ncifcrf.gov) [25, 34]. As a result, the DEGs were enriched in 11 GO terms (6 biological processes, 4 cellular components, and 1 molecular function) and one KEGG biological pathway (hsa03010: ribosome), with a significance level set at FDR $<0.05$. The enrichment levels of those GO terms are presented in circular visualization (Fig. 3). The GO terms were enriched in many downregulated genes (Fig. 3), and most of them involved ribosomal subunits: 19 RPL genes (RPL3, RPL5, RPL6, RPL7, RPL9, RPL10A, RPL11, RPL18, RPL19, RPL21, RPL22, RPL23, RPL23A, RPL26, RPL27, RPL29, RPL32, RPL35, and RPL36AL), 12 RPS genes (RPS3, RPS3A, RPS4Y1, RPS5, RPS6, RPS8, RPS11, RPS12, RPS14, RPS18, RPS24, and RPS29), and 3 MRP genes (MRPS5, MRPL16, and MRPL47).

\section{Network-based meta-analysis}

In addition to GSEA, we performed a protein-protein interaction (PPI) network analysis based on the DEGs by using NetworkAnalyst [36] (http://www.networkanalyst. ca) with the STRING Interactome database [37]. As a result, we obtained a PPI network comprising 4,164 nodes and 11,886 edges. To prune the network to a more manageable size, we conducted a zero-order interaction network analysis and detected a network containing 161 nodes and 700 edges (Fig. 4). The most highly ranked hub genes were recognized in terms of network topology measures of degree (DC) and betweenness of centrality (BC). The top-ranked 10 hub genes were EEF2 (eukaryotic elongation factor $2, \mathrm{DC}=38, \mathrm{BC}=883.9$, $\mathrm{FC}=1.22, \mathrm{FDR}=0.048)$ and 9 ribosomal proteins: $3 \mathrm{RPL}$ genes (RPL5, RPL7, and RPL23A) and 6 RPS genes (RPS3, RPS3A, RPS5, RPS6, RPS12, and RPS24) (Table 2). Many of the identified genes were common to those obtained through GSEA.

\section{Validation of potential biomarkers of AD in blood}

We examined whether many of the top-ranked hub genes could be potential blood biomarkers for AD. For this purpose, two-thirds of all samples were used as a training data set (240 samples: $180 \mathrm{ADs}$ and $60 \mathrm{CNs}$ ), and the remaining one-third was used as a test data set (122 samples: $91 \mathrm{ADs}$ and $31 \mathrm{CNs}$ ). The top-ranked $p$ hub genes were selected stepwise. A risk prediction model was constructed by using clinical information (age, sex, and $A P O E \varepsilon_{4}$ genotypes), the proportion of neutrophils, and the top-ranked $p$ hub genes with a random forest classifier using the training data. The adjusted model was then evaluated on the independent test

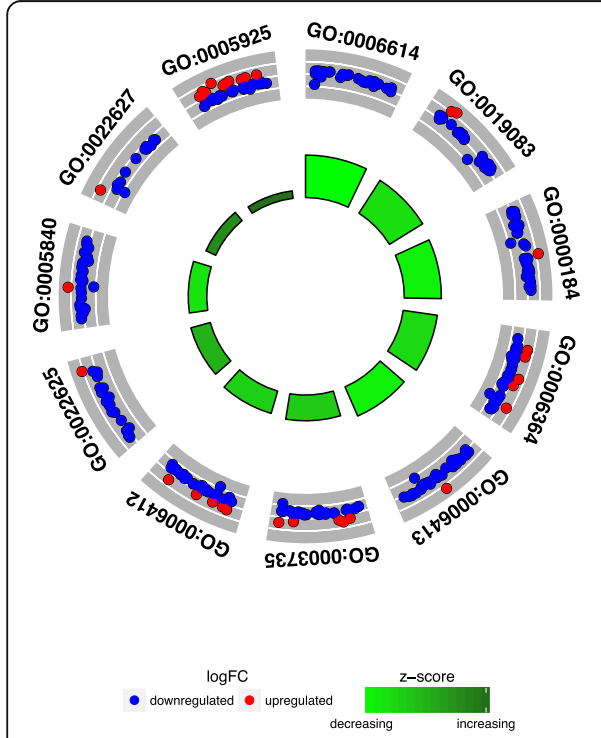

\begin{tabular}{|c|c|c|c|}
\hline ID & Description & Count & FDR(BH) \\
\hline GO:0006614 & SRP-dependent cotranslational protein targeting to membrane & 31 & 1.7E-16 \\
\hline GO:0019083 & viral transcription & 32 & $2.2 \mathrm{E}-15$ \\
\hline GO:0000184 & $\begin{array}{l}\text { nuclear-transcribed mRNA catabolic process, } \\
\text { nonsense-mediated decay }\end{array}$ & 32 & $9.9 \mathrm{E}-15$ \\
\hline GO:0006364 & rRNA processing & 41 & $6.9 \mathrm{E}-14$ \\
\hline GO:0006413 & translational initiation & 32 & $5.0 \mathrm{E}-13$ \\
\hline GO:0003735 & structural constituent of ribosome & 38 & 4.7E-11 \\
\hline GO:0006412 & translation & 39 & $2.9 \mathrm{E}-10$ \\
\hline GO:0022625 & cytosolic large ribosomal subunit & 20 & $2.9 \mathrm{E}-9$ \\
\hline GO:0005840 & ribosome & 29 & $9.6 \mathrm{E}-9$ \\
\hline GO:0022627 & cytosolic small ribosomal subunit & 14 & 3.3E-6 \\
\hline GO:0005925 & focal adhesion & 38 & 3.7E-5 \\
\hline
\end{tabular}

Fig. 3 Gene set enrichment analysis using DEGs. Statistically significant Gene Ontology terms with a false discovery rate $<0.05$ 


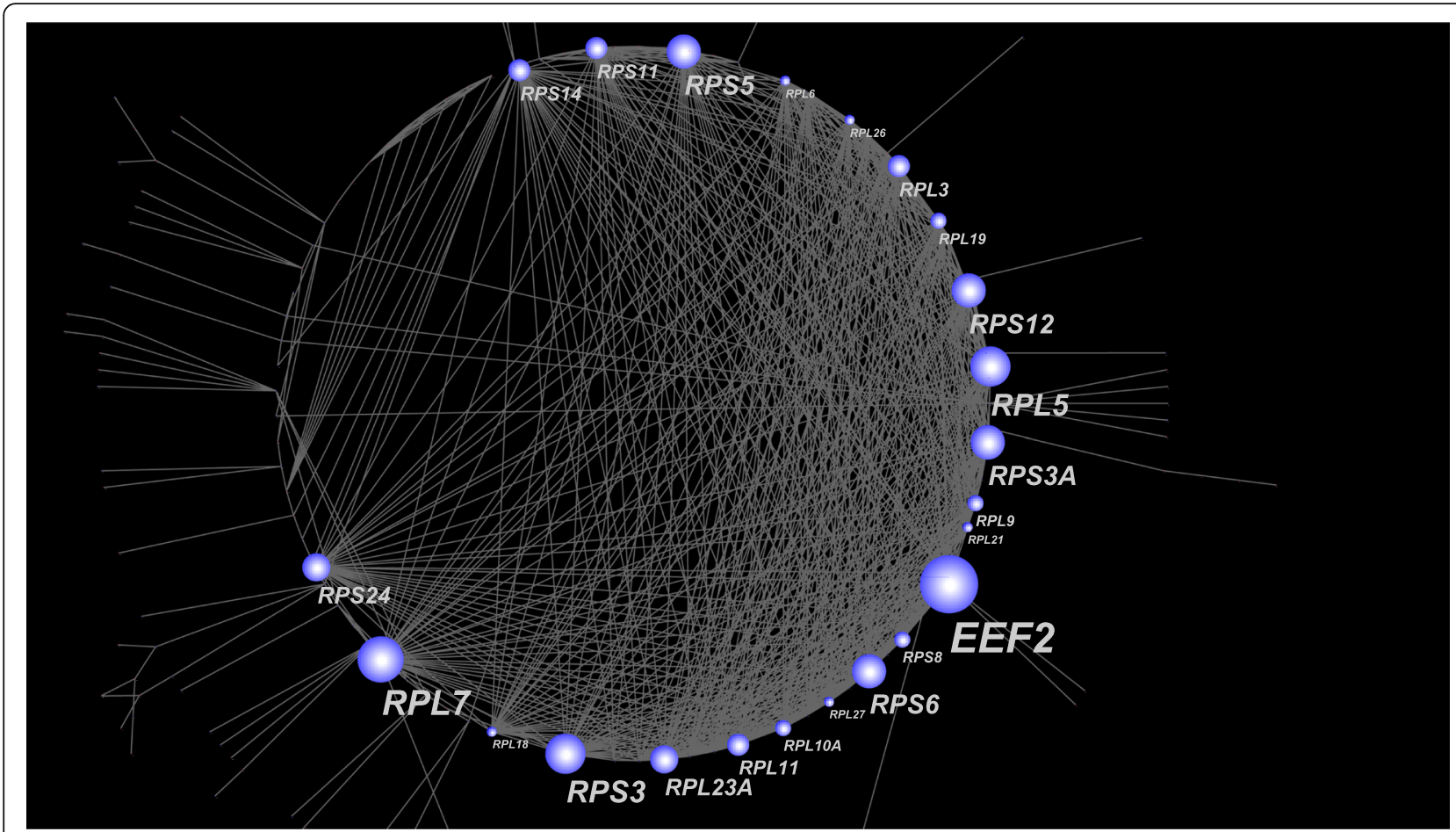

Fig. 4 Network-based meta-analysis using DEGs. A protein-protein interaction network detected in DEGs

data by using the area under the receiver operating characteristic curve (AUC). The best model achieved an AUC of 0.878 (95\% CI $0.801-0.955$, sensitivity $=0.945$, specificity $=0.710$, Supplementary Fig. S1a) in the test data when two top-ranked hub genes (EEF2 and RPL7, Fig. $5 \mathrm{a})$ were used. The highest variable importance was age $\quad$ (MeanDecreaseGini $=30.97 ; \quad R P L 7,20.63 ;$ Neut, 14.23; EEF2, 14.20; APOE $\varepsilon_{4}$ genotypes, 5.05; sex, 3.15). The best model had a superior AUC to the model using only clinical information (Fig. 5a). Furthermore, the

Table 2 Top-ranked 10 hub genes detected in the networkbased meta-analysis using DEGs

\begin{tabular}{lllll}
\hline Gene name & DC & BC & FC & FDR \\
\hline EEF2 & 38 & 883.9 & 1.22 & $4.80 \times 10^{-3}$ \\
RPL7 & 36 & 350.3 & 1.51 & $1.75 \times 10^{-5}$ \\
RPL5 & 35 & 4567.0 & 1.23 & $2.07 \times 10^{-5}$ \\
RPS3 & 35 & 1599.7 & 1.38 & $1.89 \times 10^{-4}$ \\
RPS5 & 34 & 741.0 & 1.35 & 0.024 \\
RPS12 & 34 & 427.3 & 1.44 & $5.20 \times 10^{-4}$ \\
RPS3A & 34 & 23.1 & 1.31 & $1.10 \times 10^{-3}$ \\
RPS6 & 34 & 23.1 & 1.23 & $3.31 \times 10^{-5}$ \\
RPL23A & 33 & 48.1 & 1.27 & $8.50 \times 10^{-3}$ \\
RPS24 & 33 & 22.0 & 1.23 & $8.00 \times 10^{-4}$ \\
\hline
\end{tabular}

The most highly ranked hub genes in terms of network topology measures of degree (DC) and betweenness of centrality (BC)

$F C$ fold change, FDR false discovery rate expression of two hub genes, EEF2 and RPL7, were associated with a significant decrease and increase in $A D$ prognosis, respectively $(P=0.015$ in EEF2, $P=0.032$ in $R P L 7$, Jonckheere-Terpstra trend test, Fig. $5 \mathrm{~b}$ and Supplementary Table S5). These results suggested that these two hub genes could serve as potential diagnostic blood biomarkers of AD. In a similar way, risk prediction models were constructed by using clinical features (age, sex, and $A P O E \varepsilon_{4}$ genotypes), the proportion of neutrophils, and the top-ranked two hub genes with a random forest classifier using the training data. The adjusted models were then evaluated on the independent test data for a $\mathrm{MCI}$ and $\mathrm{CN}$ set and a MCI and $\mathrm{AD}$ set. The best models achieved an AUC of $0.683(95 \% \mathrm{CI}=0.559-0.807$, sensitivity $=0.744$, specificity $=0.633$, Supplementary Fig. S1b) and an AUC of $0.645(95 \%$ CI $0.562-0.728$, sensitivity $=0.622$, specificity $=$ 0.671, Supplementary Fig. S1c) for the MCI and CN set and the $\mathrm{MCI}$ and $\mathrm{AD}$ set in the test data, respectively.

\section{Validation in a prospective cohort}

We measured mRNA expression in 248 MCI samples. Of them, $55 \mathrm{MCI}$ samples were obtained from the prospective data; 17 patients who contributed samples progressed to $\mathrm{AD}$, whereas 38 of the patients corresponding to these samples have not yet been diagnosed with bona fide $\mathrm{AD}$ after at least 1 year. Our risk prediction model based on clinical information (age, sex, and APOE $\varepsilon_{4}$ genotypes) and three potential biomarkers we obtained 
(a)

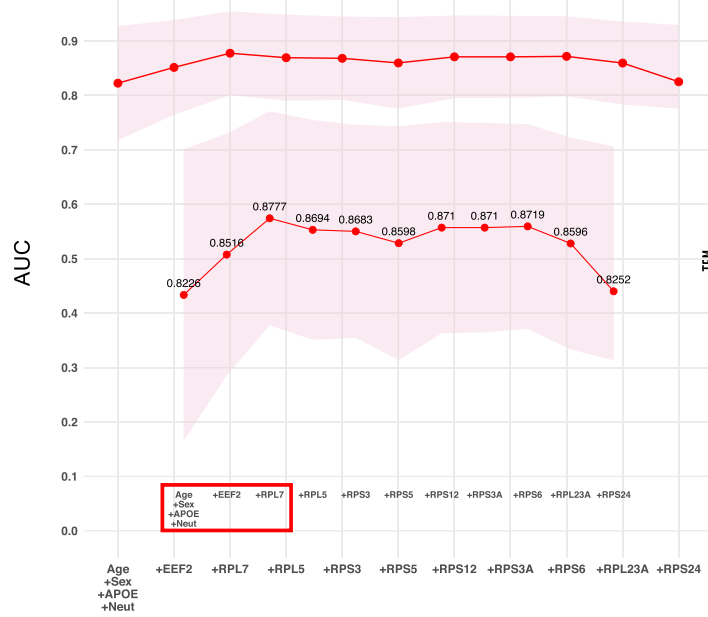

(b)

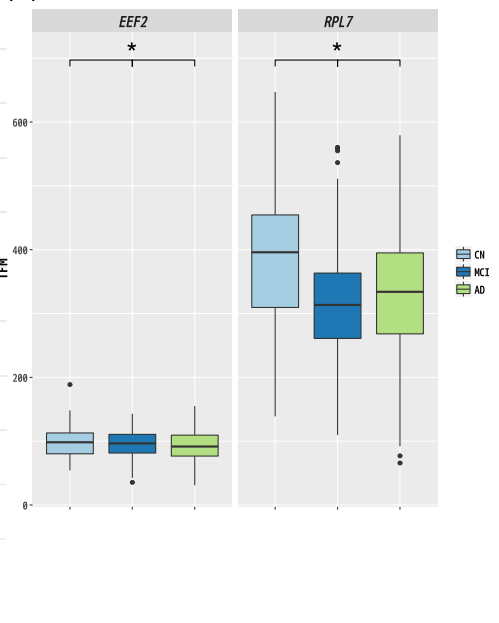

Fig. 5 Potential biomarkers of $A D$ in the blood by using the most important hub genes. a Identification of the most important hub genes by using a random forest classifier. Neut, neutrophils. b Expression of two hub genes (EEF2 and RPL7) among AD, MCI and CN samples

(i.e., the proportion of neutrophils, EEF2, and RPL7) was applied to the prospective data. Because our prediction model provides a probability of AD conversion for each MCI sample, we set the MCIs at the probability of $>0.9$ for conversion to AD. Survival probabilities were calculated by using the Kaplan-Meier method in the survival package (version 2.41.3) for the statistical software R. Our risk prediction model significantly classified the MCI samples into two categories (high and low risk). The Kaplan-Meier curves showed improved outcome for $\mathrm{AD}$ conversion-free survival (Fig. 6, log rank trend test $=0.039$ ), which achieved a high accuracy of 0.727 on the prospective cohort (sensitivity $=0.706$, specificity $=$ 0.737). Our present model predicted that 33 samples would not covert to $\mathrm{AD}$, of which 5 did convert (negative predictive value $(\mathrm{NPV})=0.848)$. Although this clear classification of samples might be helpful for future practical use in healthcare, we would have to follow those samples to improve the further predictive value.

\section{Verification of quantitative RT-PCR assay}

To validate the RNA-seq results, we used quantitative RT-PCR (qRT-PCR) analysis to evaluate the two most significant hub genes (EEF2 and RPL7) as potential biomarkers of $\mathrm{AD}$ for early diagnosis, three upregulated DEGs (TRIOBP, TAS2R39, and BASP1), and three downregulated DEGs $(L D H B, N R 1 D 2$, and PDK4). Figure 7 summarizes the RNA-seq and qRT-PCR results. Although the 8 DEGs were not expressed at precisely the same levels in both RNA-seq and qRT-PCR analyses, the regulated trends of the 8 DEGs were entirely consistent (Fig. 7). These results demonstrated our RNA-seq data accurately estimates gene expression.

\section{Discussion}

Peripheral blood biomarkers for early diagnosis have been examined in many diseases including $\mathrm{AD}$ [40-42]. In addition, various blood biomarkers associated with neurocognitive impairments have been reported, for example, glucose [43-45] and atherogenic index of plasma (AIP) [46]. However, no reliable and sensitive blood biomarkers are routinely used in clinical practice yet. One powerful and widely used approach to detect blood-based biomarkers, next-generation RNA-seq in human PBMCs, allows a comprehensive analysis of the entire transcriptome, but many of the previous studies were conducted in a small number of samples, particularly for AD.

Here, we performed comprehensive RNA-seq analysis using a large number of samples, to detect potential blood-based biomarkers associated with early diagnosis of AD. First, we used the bulk RNA-seq data to evaluate the difference in cell-type composition among $\mathrm{AD}, \mathrm{MCI}$, and $\mathrm{CN}$ samples. Of the 12 major immune cell types (B cells, plasma cells, $\mathrm{CD}^{+}{ }^{+} \mathrm{T}$ cells, $\mathrm{CD} 4^{+} \mathrm{T}$ cells, $\gamma \delta \mathrm{T}$ cells, NK cells, monocytes, macrophages, dendritic cells, mast cells, eosinophils, and neutrophils), we found a statistically significant difference in the proportion of neutrophils; that is, an increase in the proportion of neutrophils was significantly associated with $\mathrm{AD}$ prognosis. In addition, the association of this increase with prognosis was further confirmed using a large number of additional samples obtained from routine blood tests. Although a recent report suggested that the neutrophil phenotype could be associated with the rate of cognitive decline and therefore might be a prognostic blood biomarker in patients with $\mathrm{AD}$ [47], the study involved only a few samples $(n=42)$. In contrast, our current results were obtained from not only different data sets (RNA-seq and routine blood tests) but also a far 
Strata + High risk + Low risk

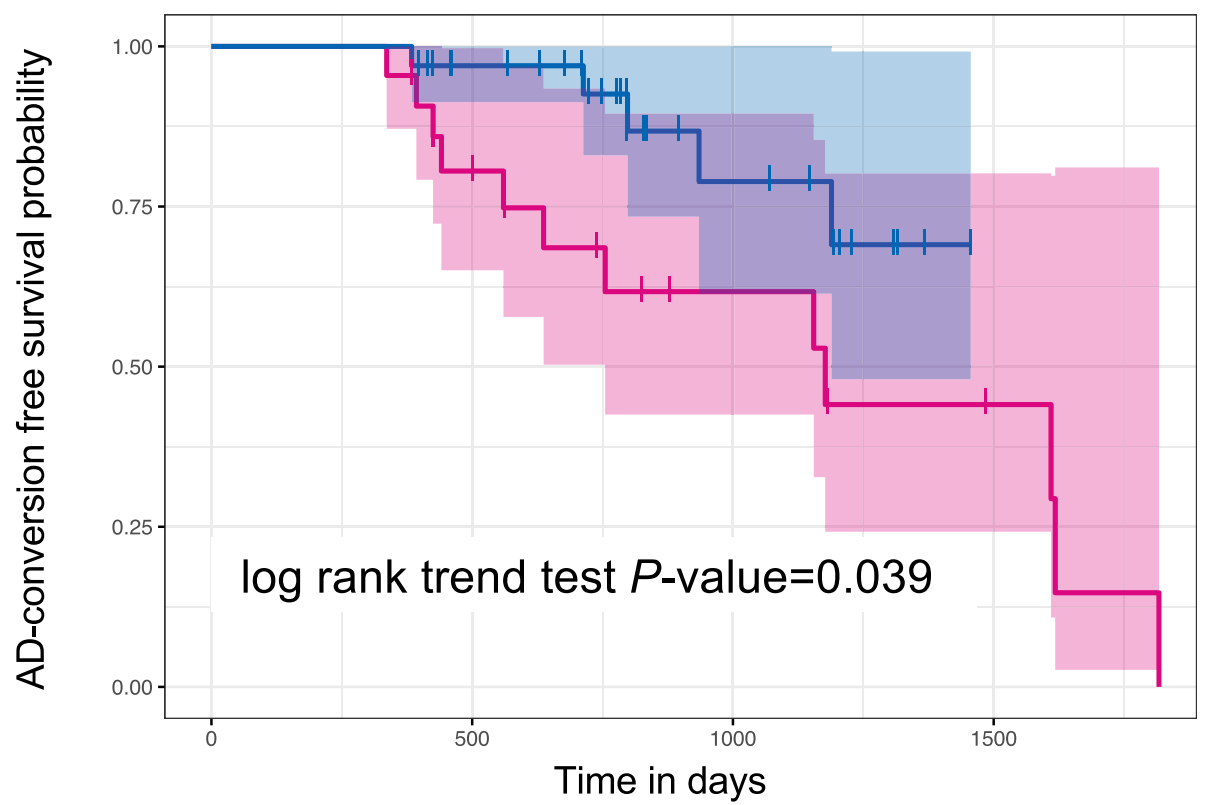

Number at risk

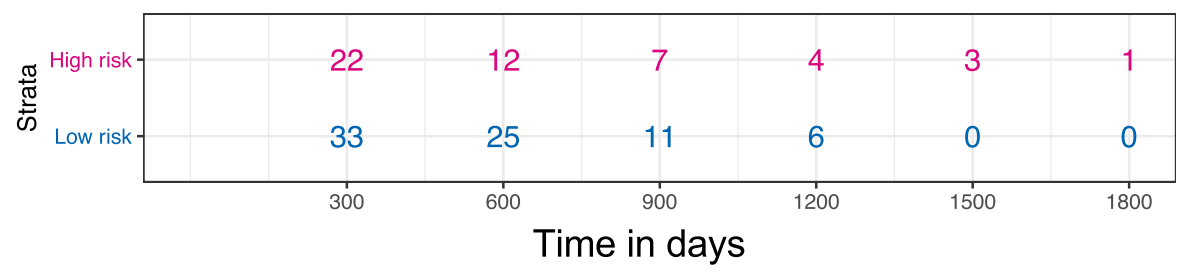

Fig. 6 Validation of potential biomarkers by using a prospective cohort

larger sample population $(n=3,099)$, providing stronger evidence that the proportion of neutrophils has the potential to be a blood biomarker of AD.

We also examined the DEGs between $\mathrm{AD}$ and $\mathrm{CN}$ samples. Of the 846 total statistically significant DEGs identified, 480 genes were upregulated and 366 were downregulated in $\mathrm{AD}$. To gain further insight into the biological functions of the identified DEGs, we performed GSEA and PPI network analysis. Multiple statistically significant GO terms, one KEGG biological pathway, and several important hub genes were identified. A risk prediction model using the top two hub genes (EEF2 and RPL7) and the proportion of neutrophils increased the model's AUC, compared with that of a model using clinical information only. Therefore, our model provides an effective and precise prediction of AD risk.

One of the potential biomarkers, EEF2, is a member of the GTP-binding translation elongation factor family and an essential factor for protein synthesis and cell survival. In recent studies, EEF2 kinase reduction alleviated $\mathrm{AD}$-associated defects in $\mathrm{AD}$ model mice [48]. In addition, $R P L 7$ is reported to be a tau-dependent $\mathrm{T}$ cell intracellular antigen 1 (TIA1)-interacting protein [49, 50]. TIA1 co-localizes with neuropathology in brain tissue of subjects with $\mathrm{AD}$, frontotemporal lobar dementia, and amyotrophic lateral sclerosis, as well as in animal models of these diseases [51-53], all of which are associated with pathological tau misfolding and aggregation. These results suggest that these two hub genes could play a key role in the pathogenesis of AD.

We applied our risk prediction model-constructed by using these three potential biomarkers (proportion of neutrophils, EEF2, and RPL7) and three clinical features (age, sex, and $A P O E \varepsilon_{4}$ genotypes)-to prospective cohort data. Although the highest variable importance was age among the six features, the three potential biomarkers interestingly showed a higher variable importance than the other clinical features (age and $A P O E \varepsilon_{4}$ genotypes). In general, when a risk prediction model is constructed by using $\mathrm{AD}$ and $\mathrm{CN}$ samples, it is difficult to apply to MCI samples. However, because our prediction model provides a probability of $\mathrm{AD}$ conversion for each sample, we were able to make it applicable to MCI samples simply by adjusting the cutoff probability for conversion. Our risk prediction model significantly 


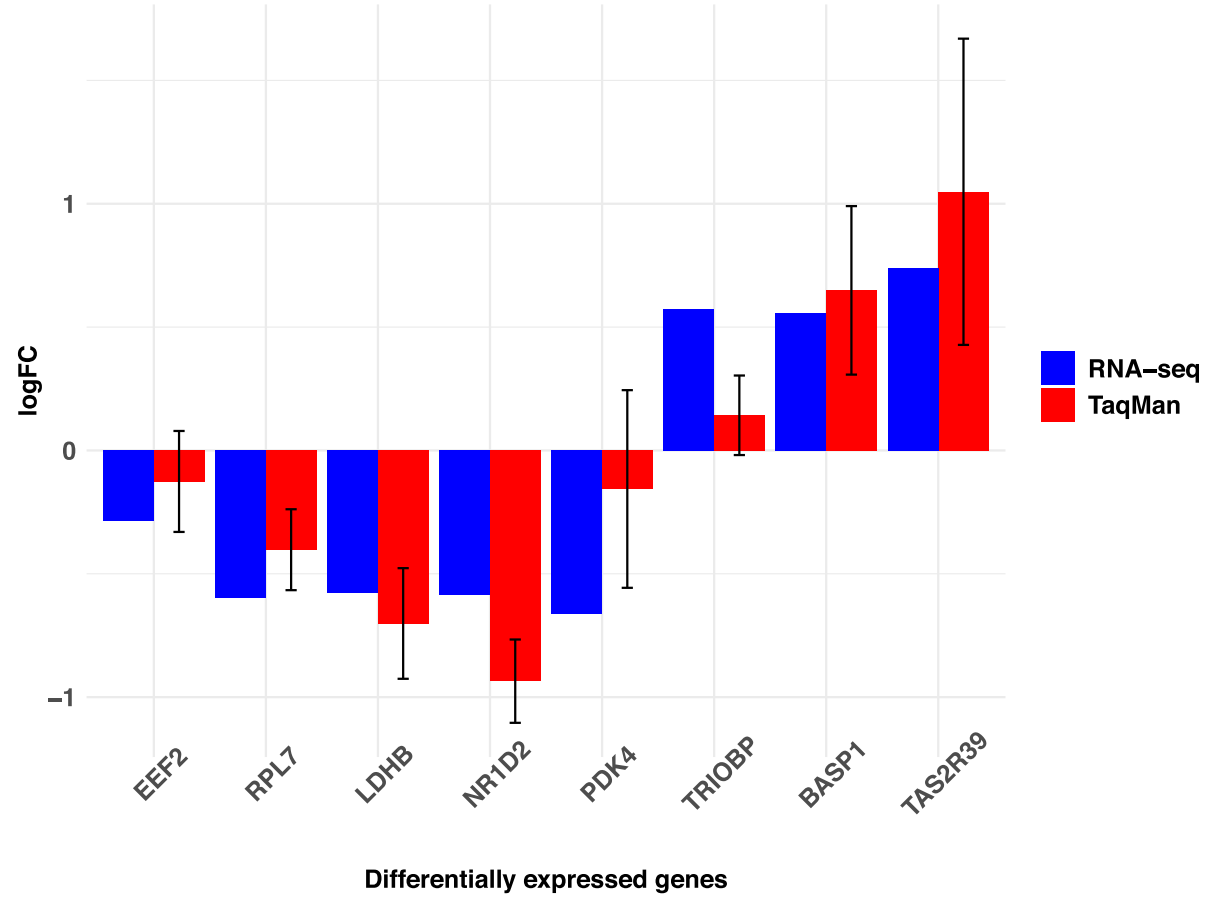

Fig. 7 qRT-PCR verification of DEGs detected by RNA-seq. Fold change values obtained from RNA-seq data: TRIOBP, TAS2R39, and BASP1 were upregulated, and EEF2, RPL7, LDHB, NR1D2, and PDK4 were downregulated. The expression determined by qRT-PCR was similar to that obtained by RNA-seq. Error bars in qRT-PCR indicate the standard error

classified MCI samples into two categories (high and low risks) and yielded a high NPV of 0.848. For clinical use, this prospective prediction model must have high NPV because it likely will be used at the first screening for $\mathrm{AD}$ conversion. This risk prediction model requires further refinement before its practical use in healthcare. One improvement would be to consider genetic variations, such as single-nucleotide variants, short insertions and deletions, and copy number variations, because GWAS have revealed many types of genetic variation that contribute to AD risk [6-8] In addition, the combination of genetic variation and gene expression-expression quantitative trait loci (eQTLs) [54-56], which are genetic variants that affect gene expression levelsshould be considered for the improvement of AD risk prediction models. Integration of that genetic variation, along with eQTL effects, likely will further improve the prospective $\mathrm{AD}$ risk prediction model.

\section{Conclusions}

The current study identified potential biomarkers for early diagnosis of AD from RNA sequencing data. The risk prediction model constructed by using the biomarkers achieved a high AUC for a validation cohort; when further applied to a prospective cohort, the model achieved high accuracy. Our model was demonstrated to be effective in prospective $\mathrm{AD}$ risk prediction. These findings indicate the discovery of potential biomarkers for early diagnosis of $\mathrm{AD}$, and their further improvement may lead to future practical clinical use.

\section{Supplementary information}

Supplementary information accompanies this paper at https://doi.org/10 1186/s13195-020-00654-x.

Additional file 1:. Supplementary Table S1. Clinical information of samples used in RNA-seq data.

Additional file 2:. Supplementary Table S2. Clinical information of samples used in routine blood tests.

Additional file 3:. Supplementary Table S3. RNA-seq data.

Additional file 4:. Supplementary Table S4. A list of all detailed DEGs.

Additional file 5: Supplementary Table S5. TPM of RPL7 and EEF2.

Additional file 6:. Supplementary Figure S1. Risk prediction models constructed using clinical information and two hub genes expression. The ROC curves of our risk prediction models in a test set. (a) AUC $=$ 0.878 in $A D$ and $C N$ (b) $A \cup C=0.683$ in $\mathrm{MCl}$ and $C N$ (c) $A \cup C=0.645$ in $\mathrm{MCl}$ and $\mathrm{AD}$

Authors' contributions

D.S. developed the method and performed the analyses. T.M. performed the experiments on mRNA expression. S.A. and S.H. provided technical assistance. H.W., T.S., and S.N. contributed to data acquisition and analyses. D.S. wrote the manuscript. D.S. and K.O. organized this work. All authors contributed to and approved the final manuscript.

\section{Funding}

This study was supported by The Japan Foundation for Aging and Health and Takeda Science Foundation (to D.S.), Research Funding for Longevity 
Sciences (29-45) from the National Center for Geriatrics and Gerontology (to K.O.), and a grant for Research on Dementia from the Japanese Ministry of Health, Labor, and Welfare (to K.O.).

\section{Availability of data and materials}

All datasets used or analyzed in the current study are available from the corresponding author on reasonable request.

\section{Ethics approval and consent to participate}

This study protocol was approved by the ethics committee of the National Center for Geriatrics and Gerontology (NCGG) of Japan. The design and performance of the current study involving human subjects were clearly described in a research protocol. All participants were volunteers and completed informed consent in writing before registering to the NCGG Biobank.

\section{Consent for publication}

Not applicable

\section{Competing interests}

The authors declare that they have no competing interests.

\section{Author details}

Medical Genome Center, National Center for Geriatrics and Gerontology, 7-430 Morioka-cho, Obu 474-8511, Aichi, Japan. ${ }^{2}$ Department of Medical Science Mathematics, Medical Research Institute, Tokyo Medical and Dental University (TMDU), Tokyo 113-8510, Japan. ${ }^{3}$ RIKEN Center for Integrative Medical Sciences, Yokohama 230-0045, Kanagawa, Japan. ${ }^{4}$ The Center for Comprehensive Care and Research on Memory Disorders, National Center for Geriatrics and Gerontology, Obu 474-8511, Aichi, Japan. ${ }^{5}$ Department of Cognitive and Behavioral Science, Nagoya University Graduate School of Medicine, Nagoya 466-8550, Aichi, Japan.

Received: 16 February 2020 Accepted: 8 July 2020

Published online: 16 July 2020

\section{References}

1. Robinson L, Tang E, Taylor JP. Dementia: timely diagnosis and early intervention. BMJ. 2015;350:h3029.

2. Kim DH, Yeo SH, Park JM, Choi JY, Lee TH, Park SY, Ock MS, Eo J, Kim HS, Cha HJ. Genetic markers for diagnosis and pathogenesis of Alzheimer's disease. Gene. 2014;545:185-93.

3. Zetterberg H. Applying fluid biomarkers to Alzheimer's disease. Am J Physiol Cell Physiol. 2017;313:C3-C10

4. Zverova M. Alzheimer's disease and blood-based biomarkers - potential contexts of use. Neuropsychiatr Dis Treat. 2018;14:1877-82.

5. Ashraf GM, Chibber S, Mohammad, Zaidi SK, Tabrez S, Ahmad A, Shakil S, Mushtaq G, Baeesa SS, Kamal MA. Recent updates on the association between Alzheimer's disease and vascular dementia. Med Chem. 2016;12:226-37.

6. Jansen IE, Savage JE, Watanabe K, Bryois J, Williams DM, Steinberg S, Sealock J, Karlsson IK, Hagg S, Athanasiu L, et al. Genome-wide meta-analysis identifies new loci and functional pathways influencing Alzheimer's disease risk. Nat Genet. 2019:51:404-13.

7. Grau S, de Rojas I, Hernandez I, Quintela I, Montrreal L, Alegret M, HernandezOlasagarre B, Madrid L, Gonzalez-Perez A, Maronas O, et al. Genome-wide association analysis of dementia and its clinical endophenotypes reveal novel loci associated with Alzheimer's disease and three causality networks: The GR@ACE project. Alzheimers Dement. 2019:15:1333-47.

8. Kunkle BW, Grenier-Boley B, Sims R, Bis JC, Damotte V, Naj AC, Boland A, Vronskaya M, van der Lee SJ, Amlie-Wolf A, et al. Genetic meta-analysis of diagnosed Alzheimer's disease identifies new risk loci and implicates $A \beta$, tau, immunity and lipid processing. Nat Genet. 2019:51:414-30.

9. Albert MS, DeKosky ST, Dickson D, Dubois B, Feldman HH, Fox NC, Gamst A, Holtzman DM, Jagust WJ, Petersen RC, et al. The diagnosis of mild cognitive impairment due to Alzheimer's disease: recommendations from the National Institute on Aging-Alzheimer's Association workgroups on diagnostic guidelines for Alzheimer's disease. Alzheimers Dement. 2011;7:270-9.

10. McKhann GM, Knopman DS, Chertkow H, Hyman BT, Jack CR Jr, Kawas CH, Klunk WE, Koroshetz WJ, Manly JJ, Mayeux R, et al. The diagnosis of dementia due to Alzheimer's disease: recommendations from the National
Institute on Aging-Alzheimer's Association workgroups on diagnostic guidelines for Alzheimer's disease. Alzheimers Dement. 2011;7:263-9.

11. De Meyer G, Shapiro F, Vanderstichele H, Vanmechelen E, Engelborghs S, De Deyn PP, Coart E, Hansson O, Minthon L, Zetterberg H, et al. Diagnosisindependent Alzheimer disease biomarker signature in cognitively normal elderly people. Arch Neurol. 2010;67:949-56.

12. Fagan AM, Shaw LM, Xiong C, Vanderstichele H, Mintun MA, Trojanowski JQ, Coart E, Morris JC, Holtzman DM. Comparison of analytical platforms for cerebrospinal fluid measures of $\beta$-amyloid 1-42, total tau, and p-tau181 for identifying Alzheimer disease amyloid plaque pathology. Arch Neurol. 2011; 68:1137-44.

13. Miller G. Alzheimer's biomarker initiative hits its stride. Science. 2009;326:386-9.

14. Mistur R, Mosconi L, Santi SD, Guzman M, Li Y, Tsui W, de Leon MJ. Current challenges for the early detection of Alzheimer's disease: brain imaging and CSF studies. J Clin Neurol. 2009:5:153-66.

15. Schmand B, Eikelenboom P, van Gool WA, Alzheimer's Disease Neuroimaging I. Value of neuropsychological tests, neuroimaging, and biomarkers for diagnosing Alzheimer's disease in younger and older age cohorts. J Am Geriatr Soc. 2011:59:1705-10.

16. Mattsson N, Cullen NC, Andreasson U, Zetterberg H, Blennow K. Association between longitudinal plasma neurofilament light and neurodegeneration in patients with Alzheimer disease. JAMA Neurol. 2019;76:791-9.

17. Janelidze S, Mattsson N, Palmqvist S, Smith R, Beach TG, Serrano GE, Chai X Proctor NK, Eichenlaub U, Zetterberg $\mathrm{H}$, et al. Plasma P-tau181 in Alzheimer's disease: relationship to other biomarkers, differential diagnosis, neuropathology and longitudinal progression to Alzheimer's dementia. Nat Med. 2020;26:379-86.

18. Liu T, Yu N, Ding F, Wang S, Li S, Zhang X, Sun X, Chen Y, Liu P. Verifying the markers of ovarian cancer using RNA-seq data. Mol Med Rep. 2015;12:1125-30.

19. Wang H, Li Y, Ryder JW, Hole JT, Ebert PJ, Airey DC, Qian HR, Logsdon B, Fisher A, Ahmed Z, et al. Genome-wide RNAseq study of the molecular mechanisms underlying microglia activation in response to pathological tau perturbation in the rTg4510 tau transgenic animal model. Mol Neurodegener. 2018:13:65.

20. Bennett JP Jr, Keeney PM, Brohawn DG. RNA sequencing reveals small and variable contributions of infectious agents to transcriptomes of postmortem nervous tissues from amyotrophic lateral sclerosis, Alzheimer's disease and Parkinson's disease subjects, and increased expression of genes from disease-activated microglia. Front Neurosci. 2019;13:235.

21. Anders S, Huber W. Differential expression analysis for sequence count data. Genome Biol. 2010:11:R106.

22. Li J, Tibshirani R. Finding consistent patterns: a nonparametric approach for identifying differential expression in RNA-Seq data. Stat Methods Med Res. 2013;22:519-36

23. Zhu Q, Sun Y, Zhou Q, He Q, Qian H. Identification of key genes and pathways by bioinformatics analysis with TCGA RNA sequencing data in hepatocellular carcinoma. Mol Clin Oncol. 2018:9:597-606.

24. Ashburner M, Ball CA, Blake JA, Botstein D, Butler H, Cherry JM, Davis AP, Dolinski K, Dwight SS, Eppig JT, et al. Gene ontology: tool for the unification of biology. The Gene Ontology Consortium. Nat Genet. 2000;25:25-9.

25. Huang da W, Sherman BT, Lempicki RA: Bioinformatics enrichment tools: paths toward the comprehensive functional analysis of large gene lists. Nucleic Acids Res 2009, 37:1-13.

26. Kanehisa M, Furumichi M, Tanabe M, Sato Y, Morishima K. KEGG: new perspectives on genomes, pathways, diseases and drugs. Nucleic Acids Res. 2017:45:D353-61.

27. Kanehisa M, Sato Y, Furumichi M, Morishima K, Tanabe M. New approach for understanding genome variations in KEGG. Nucleic Acids Res. 2019;47:D590-5.

28. Shigemizu D, Akiyama S, Asanomi Y, Boroevich KA, Sharma A, Tsunoda T, Matsukuma K, Ichikawa M, Sudo H, Takizawa S, et al. Risk prediction models for dementia constructed by supervised principal component analysis using miRNA expression data. Commun Biol. 2019:2:77.

29. Dobin A, Davis CA, Schlesinger F, Drenkow J, Zaleski C, Jha S, Batut P, Chaisson M, Gingeras TR. STAR: ultrafast universal RNA-seq aligner. Bioinformatics. 2013;29:15-21.

30. Liao Y, Smyth GK, Shi W. featureCounts: an efficient general purpose program for assigning sequence reads to genomic features. Bioinformatics. 2014;30:923-30.

31. Robinson MD, McCarthy DJ, Smyth GK. edgeR: a Bioconductor package for differential expression analysis of digital gene expression data. Bioinformatics. 2010;26:139-40. 
32. Li B, Dewey CN. RSEM: accurate transcript quantification from RNA-Seq data with or without a reference genome. BMC Bioinformatics. 2011;12:323.

33. Chen B, Khodadoust MS, Liu CL, Newman AM, Alizadeh AA. Profiling tumor infiltrating immune cells with CIBERSORT. Methods Mol Biol. 2018;1711:243-59.

34. Huang da W, Sherman BT, Lempicki RA: Systematic and integrative analysis of large gene lists using DAVID bioinformatics resources. Nat Protoc 2009, 4:44-57.

35. Walter W, Sanchez-Cabo F, Ricote M. GOplot: an R package for visually combining expression data with functional analysis. Bioinformatics. 2015;31: 2912-4.

36. Santiago JA, Potashkin JA. Network-based metaanalysis identifies HNF4A and PTBP1 as longitudinally dynamic biomarkers for Parkinson's disease. Proc Natl Acad Sci U S A. 2015;112:2257-62.

37. Szklarczyk D, Franceschini A, Kuhn M, Simonovic M, Roth A, Minguez $P$, Doerks T, Stark M, Muller J, Bork P, et al. The STRING database in 2011: functional interaction networks of proteins, globally integrated and scored. Nucleic Acids Res. 2011;39:D561-8.

38. Su G, Morris JH, Demchak B, Bader GD: Biological network exploration with Cytoscape 3. Curr Protoc Bioinformatics 2014, 47:8 13 11-24.

39. Marone M, Mozzetti S, De Ritis D, Pierelli L, Scambia G. Semiquantitative RTPCR analysis to assess the expression levels of multiple transcripts from the same sample. Biol Proced Online. 2001;3:19-25.

40. Long J, Pan G, Ifeachor E, Belshaw R, Li X. Discovery of novel biomarkers for Alzheimer's disease from blood. Dis Markers. 2016;2016:4250480.

41. Rai N, Kumar R, Desai GR, Venugopalan G, Shekhar S, Chatterjee P, Tripathi M, Upadhyay AD, Dwivedi S, Dey AB, Dey S. Relative alterations in bloodbased levels of sestrin in Alzheimer's disease and mild cognitive impairment patients. J Alzheimers Dis. 2016;54:1147-55.

42. San Segundo-Acosta P, Montero-Calle A, Fuentes M, Rabano A, Villalba M, Barderas R. Identification of Alzheimer's disease autoantibodies and their target biomarkers by phage microarrays. J Proteome Res. 2019;18:2940-53.

43. Grober E, Hall CB, Hahn SR, Lipton RB. Memory impairment and executive dysfunction are associated with inadequately controlled diabetes in older adults. J Prim Care Community Health. 2011;2:229-33.

44. Crane PK, Walker R, Hubbard RA, Li G, Nathan DM, Zheng H, Haneuse S, Craft S, Montine TJ, Kahn SE, et al. Glucose levels and risk of dementia. N Engl J Med. 2013;369:540-8.

45. Pappas C, Andel R, Infurna FJ, Seetharaman S. Glycated haemoglobin (HbA1c), diabetes and trajectories of change in episodic memory performance. J Epidemiol Community Health. 2017;71:115-20.

46. Aniwattanapong D, Tangwongchai S, Supasitthumrong T, Hemrunroj S, Tunvirachaisakul C, Tawankanjanachot I, Chuchuen P, Snabboon T, Carvalho AF, Maes M. Validation of the Thai version of the short Boston Naming Test (TBNT) in patients with Alzheimer's dementia and mild cognitive impairment: clinical and biomarker correlates. Aging Ment Health. 2019;23:840-50.

47. Dong Y, Lagarde J, Xicota L, Corne H, Chantran Y, Chaigneau T, Crestani B, Bottlaender M, Potier MC, Aucouturier P, et al. Neutrophil hyperactivation correlates with Alzheimer's disease progression. Ann Neurol. 2018;83:387-405.

48. Beckelman BC, Yang W, Kasica NP, Zimmermann HR, Zhou X, Keene CD, Ryazanov AG, Ma T. Genetic reduction of eEF2 kinase alleviates pathophysiology in Alzheimer's disease model mice. J Clin Invest. 2019;129:820-33.

49. Minjarez B, Valero Rustarazo ML, Sanchez del Pino MM, Gonzalez-Robles A, Sosa-Melgarejo JA, Luna-Munoz J, Mena R, Luna-Arias JP. Identification of polypeptides in neurofibrillary tangles and total homogenates of brains with Alzheimer's disease by tandem mass spectrometry. J Alzheimers Dis. 2013:34:239-62.

50. Vanderweyde T, Apicco DJ, Youmans-Kidder K, Ash PEA, Cook C, Lummertz da Rocha E, Jansen-West K, Frame AA, Citro A, Leszyk JD, et al. Interaction of tau with the RNA-binding protein TIA1 regulates tau pathophysiology and toxicity. Cell Rep. 2016;15:1455-66.

51. Liu-Yesucevitz L, Bilgutay A, Zhang YJ, Vanderweyde T, Citro A, Mehta T, Zaarur N, McKee A, Bowser R, Sherman M, et al. Tar DNA binding protein-43 (TDP-43) associates with stress granules: analysis of cultured cells and pathological brain tissue. PLoS One. 2010;5:e13250.

52. Thomas MG, Loschi M, Desbats MA, Boccaccio GL. RNA granules: the good, the bad and the ugly. Cell Signal. 2011;23:324-34.

53. Vanderweyde T, Yu H, Varnum M, Liu-Yesucevitz L, Citro A, Ikezu T, Duff K Wolozin B. Contrasting pathology of the stress granule proteins TIA-1 and G3BP in tauopathies. J Neurosci. 2012;32:8270-83.

54. Schadt EE, Monks SA, Drake TA, Lusis AJ, Che N, Colinayo V, Ruff TG, Milligan SB, Lamb JR, Cavet G, et al. Genetics of gene expression surveyed in maize, mouse and man. Nature. 2003;422:297-302.
55. Stranger BE, Forrest MS, Clark AG, Minichiello MJ, Deutsch S, Lyle R, Hunt S, Kahl B, Antonarakis SE, Tavare $\mathrm{S}$, et al. Genome-wide associations of gene expression variation in humans. PLoS Genet. 2005;1:e78.

56. Emilsson V, Thorleifsson G, Zhang B, Leonardson AS, Zink F, Zhu J, Carlson S, Helgason A, Walters GB, Gunnarsdottir S, et al. Genetics of gene expression and its effect on disease. Nature. 2008:452:423-8.

\section{Publisher's Note}

Springer Nature remains neutral with regard to jurisdictional claims in published maps and institutional affiliations.
Ready to submit your research? Choose BMC and benefit from:

- fast, convenient online submission

- thorough peer review by experienced researchers in your field

- rapid publication on acceptance

- support for research data, including large and complex data types

- gold Open Access which fosters wider collaboration and increased citations

- maximum visibility for your research: over $100 \mathrm{M}$ website views per year

At BMC, research is always in progress.

Learn more biomedcentral.com/submissions 